\title{
Estereotipos regionales y nacionales: del conocimiento individual a la sociedad pensante
}

\author{
María Soledad Rodríguez, José Manuel Sabucedo \\ y Constantino Arce \\ Universidad de Santiago de Compostela, España.
}

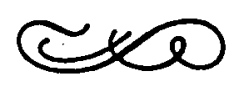

\begin{abstract}
Resumen
Este trabajo pretende descubrir la estructura subyacente a la percepción que los sujetos gallegos tienen de diferentes estereotipos regionales y nacionales (i. e. andaluces, castellanos, vascos, europeos, españoles, catalanes y gallegos). Este objetivo será contemplado desde un modelo de distancia espacial, el Escalamiento Multidimensional, que nos permitiría estudiar la distancia psicológica entre los diferentes grupos, en la configuración espacial ofrecida por el modelo. Los resultados apuntan a una doble dimensionalidad: competencia personal y valores sociales, a la hora de valorar a los diferentes estereotipos. Además esta dimensionalidad se mantiene a través de las distintas submuestras utilizadas: orientación política de derecha vs. de izquierda, universitarios - no universitarios, hombres- mujeres, dando cuenta de la estabilidad de los estereotipos, y de la dimensión social de los mismos.
\end{abstract}

Palabras clave: Estereotipos nacionales.

\section{Regional and National Stereotypes: From Individual Knowledge to the Thinking Society}

\begin{abstract}
The main purpose of this study is to uncover the underlying structure of subjects' perception of several regional and national stereotypes. The study is carried out from a proximity data approach. Stimuli are mapped as points in space by means of multidimensional scaling techniques. The two-dimensional solution was retained, the first one being interpreted as a "personal competences" dimension, and the second one as a "social values" dimension. The stability of the configuration was obtained throughout various subgroups (males vs. females, left wing subjects vs. right wing subjects, and bigh educational level vs. low or medium educational level).
\end{abstract}

Keywords: National stereotypes.

Dirección del autor: Separatas del artículo pueden solicitarse a cualquiera de los autores, Facultad de Psicología, Universidad de Santiago de Compostela, España. 
Existe un acuerdo unánime entre todos los estudiosos del tema respecto al momento de aparición del término «estereotipo» en las ciencias sociales. Efectivamente, sería W. Lippmann quien en 1922 acuñaría el término para designar "esas imágenes en nuestra cabeza" ("pictures in our heads») que median entre la realidad y la percepción que nosotros tenemos de ella, provocando una simplificación o una orientación selectiva de nuestras percepciones que podrían conducir a distorsiones más o menos graves de la realidad objetiva.

A partir de la obra de este autor se irían ofreciendo, a lo largo del tiempo, distintas definiciones y aproximaciones teóricas de los estereotipos. Sin embargo, como ya señalaba Brigham (1971), 50 años después de la obra de Lippmann, los autores no muestran un consenso pleno en cuanto al concepto mismo de estereotipo. Al respecto, la definición dada por Marlene Mackie (1973, p. 435) parece incluir los rasgos que se consideran esenciales de los estereotipos, al referirse a ellos como «aquellas creencias populares sobre los atributos que caracterizan a una categoría social y sobre los que hay un acuerdo sustancial». Por otra parte, aquellas definiciones que intentan especificar características y funciones de los estereotipos, no son ya sino un claro reflejo de los diferentes enfoques o perspectivas teóricas.

Actualmente, desde una perspectiva cognitiva, se asume que los estereotipos resultan de un "proceso de categorización" (Tajfel, 1984), dado que la percepción diferencial de determinados grupos de personas sería imposible sin una previa categorización de los individuos en grupos. De este modo, todos utilizamos categorías para describir a numerosos grupos de personas; y utilizamos el proceso psicológico de la categorización para ordenar y sistematizar la gran cantidad de información que recibimos del entorno. En este sentido, la categorización desempeñaría un papel práctico en la economía del pensamiento; función que explicaría la simplificación que se opera en la percepción del entorno tanto físico como social (Tajfel, 1975). Así, desde esta perspectiva, estaríamos contemplando el estereotipo en tanto que proceso, es decir la estereotipia, al concebirlo como «la tendencia a atribuir características generalizadas y simplificadas a grupos de gentes en forma de etiquetas verbales" (Vinacke, 1956). En este sentido, los estereotipos comportarían la asignación de ciertos rasgos a los individuos que son miembros de un' grupo y también la atribución de ciertas diferencias respecto a los miembros de otros grupos. En la medida en que dos o más grupos participan de la asignación en común de diferentes rasgos, podemos decir que dichos grupos son percibidos subjetivamente por los individuos como más semejantes, y en caso contrario como más distintos. En consecuencia, este proceso servirá para explicar las diferencias o semejanzas subjetivas percibidas entre los grupos.

Este énfasis en lo congnitivo, que por lo demás ya estaba presente en el trabajo pionero de Allport (1954), cuando este autor se refiere a la importancia del proceso de categorización en el desarrollo del prejuicio, tiene serias implicaciones para la aproximación al tema de los estereotipos. En la interesante reflexión que Sangrador (1990) realiza sobre la evolución de la definición de esterotipos, se señala de forma clara una de las consecuencias negativas de la asunción de los planteamientos cognitivistas: la consideración de los estereotipos como una estructura cognitiva individual. De hecho, como recuerda Sangrador, Hamilton (1981) considera a los estereotipos como «creencias de individuo sobre un grupo social». Desde esta perspectiva, pues, el estereotipo 
pierde su carácter de "compartido», y se transforma en un mero proceso cognitivo individual. Las implicaciones de todo tipo de esta orientación psicologicista en psicología social, han sido señaladas en diversidad de momentos y a propósito de muy diferentes cuestiones (Caplan y Nelson, 1973; Katz, 1974; Moscovici, 1982; Sabucedo, 1990).

Sin embargo, y aunque hablar de fronteras geográficas en la producción del conocimiento resulta a veces una simplificación excesiva, hay que reconocer que los psicólogos sociales europeos han mostrado, en relación con sus colegas norteamericanos, una mayor sensibilidad por la dimensión social en el estudio de los estereotipos. Tajfel (1984) alude a la definición de estereotipo dada por Stallybrass en The Fontana Dictionary of Modern Thought, y a las declaraciones de varios autores y directores teatrales, para poner de manifiesto el carácter compartido de los estereotipos y las funciones sociales que cumplen. Estas aproximaciones a la definición de estereotipo, brindadas por «legos» en la materia, las contrasta Tajfel con citas de eminentes psicólogos sociales como Hamilton y Guilford (1976), Taylor y col. (1978), Taylor y Aboud (1973), y del propio Tajfel (Tajfel y Wilkies, 1963), con el fin de mostrar como éstos últimos, y a diferencia de los no expertos, relegan la naturaleza social de los estereotipos. En este contexto, y a modo de antídoto contra la corriente psicologicista que proveniente allende del Océano trata de dominar el panorama de la psicología social, no está de más recordar algunos de los planteamientos del propio Tajfel sobre este tema. Para Tajfel, es el carácter compartido de los estereotipos el que hace que se pueda hablar de estereotipos sociales. Por otro lado, este autor afirma que existen dos problemas que no pueden ser resueltos desde una óptica exclusivamente cognitiva. En primer lugar, las funciones de los estereotipos; y en segundo lugar, «la naturaleza de los nexos entre estas funciones sociales o de grupo de los este eotipos y su común adopción por gran número de personas que comparten una afiliación social» (1981, pp. 173-174).

Como se desprende de lo anterior, la cuestión del carácter compartido o no de los estereotipos, no es una cuestión baladí; tiene serias consecuencias para el modo de entender este tema y nuestra propia disciplina, así como una indudable vertiente social e incluso, ipor qué no decirlo?, política.

Por lo que respecta a los trabajos empíricos sobre los estereotipos, debemos mencionar el estudio pionero de Katz y Braly (1933). Estos autores introducirían un instrumento de medida de los estereotipos: el «Adjetive Check List» que se componía de una lista de 84 adjetivos, referidos a atributos personales, que los sujetos experimentales debían atribuir a los diferentes grupos étnicos objeto de estudio. A partir de la obra de estos autores, la mayoría de las investigaciones posteriores se limitarían a recoger los rasgos más atribuidos para calificar a los diferentes grupos érnicos, con el objeto de comparar la lista de adjetivos obtenida con los resultados ofrecidos por Katz y Braly (1933): la similaridad entre ambos permitía a los autores conluir en la estabilidad de los esterotipos (Meenes, 1943; Gilbert, 1951; Karlins, Coffman y Walters, 1969; etc).

En. España sería Pinillos (1960) quien estudiaría la semejanza, en los atributos asignados a diferentes grupos étnicos, entre universitarios españoles, norteamericanos e ingleses. En la misma línea, otros autores españoles (Rodríguez Sanabra, 1963 y Chacón Fuertes, 1986) abordarían el estudio empírico de los estereoripos. 
Posteriormente, en 1981, Sangrador publicó un extenso trabajo sobre el tema que pretendía ir más allá de la simple recogida de los estereotipos que unos grupos mantenían hacia otros; al intentar lograr una estructura subyacente de los contenidos de dichos esterotipos. Para ello incluyó, en uno de los objetivos de su trabajo, al estudio de la similaridad entre los atributos referidos a los distintos grupos étnicos, por medio de un modelo de distancia no-espacial: el Análisis de Clusters.

Tómando como punto de partida el citado estudio, nuestra investigación ha pretendido abordar la misma cuestión mediante un modelo de distancia espacial: el Escalamiento Multidimensional (MDS). El MDS permite visualizar la estructura profunda de los datos, y al ser un modelo continuo, a diferencia del cluster que es discreto, posibilita una mayor precisión en el análisis de la realidad psicológica que se está analizando.

Un primer objetivo de este estudio consistió, pues, en descubrir la estructura subyacente a la percepción ciudadana de diferentes estereotipos regionales y nacionales (gallegos, castellanos, españoles, andaluces, catalanes, europeos, y vascos) utilizando datos de proximidad, en lugar de los habituales datos de perfil (Arce, 1989a). Esto nos permitirá conocer la diferencia en la percepción de los distintos exogrupos, la relación existente entre los mismos, y la distancia que mantienen respecto al endogrupo.

Un segundo objetivo fue explorar el grado en que esas representaciones son compartidas por diferentes submuestras de población. Esto nos permitiría poner de manifiesto la naturaleza social de los esterotipos.

En orden a satisfacer estos objetivos, se ha utilizado la técnica de Escalamiento Multidimensional, que nos permitiría obtener una representación de dichos grupos en un espacio n-dimensional, de tal manera que cada estereotipo sea representado por un punto en el espacio y la disimilaridad percibida entre pares de estereotipos por la distancia euclidiana entre dos puntos (Arce, 1989b; Arce, Seoane y Varela, 1989). Además, se hizo uso también del Cluster Análisis con el fin de conocer las pequeñas estructuras.

Para ello, se obtuvieron medidas directas de la disimilaridad entre pares de estímulos (i. e. estereotipos) y juicios subjetivos de las propiedades de los estímulos, que fueron utilizadas para la elección e interpretación de la dimensionalidad mínima subyacente (Arce, 1989b).

Posteriormente, en una segunda fase del estudio, se decidió contemplar la influencia de una tercera variable (i. e. actitud política) en la representación cognitiva de los estereotipos.

\section{METODO}

La Muestra de sujetos participantes fue de 86, de éstos 40 eran trabajadores (todos varones) en una empresa pública de la ciudad de La Coruña y 46 estudiantes de $4 .^{\circ}$ y $50^{\circ}$ de Psicología de la Universidad de Santiago de Compostela. De la muestra total 55 eran hombres y 31 mujeres. Su edad media era de 23,53 años y su desviación típica 1,85. La edad mínima era de 21 años y la máxima de 29 . Todos ellos llevaban viviendo la mayor parte de su vida en Galicia. Su participación fue voluntaria. Posteriormente, con el fin de contemplar el tercero de nuestros objetivos, se tomó una segunda muestra de 51 estudiantes de Psicología, de los cuales 37 se consideraban de izquierdas y 14 de derechas. 
Como estímulos fueron seleccionados los 7 grupos de habitantes de las diferentes regiones y nacionalidades ya mencionadas, es decir: andaluces, castellanos, catalanes, gallegos, vascos, españoles y europeos.

Con los 7 estímulos seleccionados se formaron 21 pares $(7 \times 6 / 2)$. Para cada par, se pidió a los sujetos que juzgaran la disimilaridad percibida entre ambos estímulos en una escala discreta de 9 puntos, donde 1 significaba muy similares y 9 muy disimilares.

Para ordenar los estímulos se utilizó el método de rotación estándar (Davison, 1983, pp. 45-47). A la mitad de los sujetos se les pasaron los pares obtenidos según este método y a la otra mitad en orden inverso. Antes de comenzar la tarea se pidió a los sujetos que leyeran todos los pares de estímulos con el fin de igualar sus expectativas.

Como el número de estímulos era pequeño $(n=7)$, los 21 pares de estímulos fueron juzgados por todos los sujetos. Así, los datos recogidos para cada sujeto (disimilaridades) quedaron comprendidos en una matríz simétrica $(7 \times 7)$ sin elementos en la diagonal principal, donde tanto las filas como las columnas eran estímulos.

Adicionalmente, los sujetos evaluaron cada estímulo independientemente en 19 escalas bipolares, discretas, de 9 puntos, que aparecen recogidas en la Tabla I.

TABLA I

Relación de adjetivos bipolares

Pacíficos / violentos

Generosos / egoístas

Confiados / desconfiados

Tradicionales / modernos

Rígidos / flexibles

Solidarios / insolidarios

Agradables / desagradables

Impulsivos / no impulsivos

Inteligentes / no inteligentes

Amantes de su tierra /

no amantes de su tierra
Tolerantes / intolerantes

Sinceros / falsos

Responsables / irresponsables

Sensibles / insensibles

Arrogantes / sencillos

Idealistas / materialistas

Próximos / distantes

Ambiciosos / modestos

Trabajadores / no trabajadores

Tanto los órdenes de los estímulos como los de las escalas bipolares fueron balanceados. Los sujetos que constituían la segunda muestra debían además emitir un juicio sobre su actitúd política, en una escala discreta de 7 puntos, donde 1 significaba totalmente a la izquierda y 7 totalmente a la derecha. Solamente se tendrían en cuenta en el estudio aquellos sujetos que señalaban como puntuación en la escala los números $1,2,6$, y 7 , con el fin de excluir aquellos que se situaban en posiciones intermedias.

Los adjetivos mencionados fueron seleccionados en función de un estudio piloto previo. Para ello, se pidió a 10 sujetos elegidos al azar (todos ellos estudiantes y profesores de Psicología de la Universidad de Santiago de Compostela) que nos mencionaran adjetivos que, a su juicio, servían para describir a los habitantes de diferentes regiones y nacionalidades. Dado que el número de estímulos encontrados fue muy elevado, reducimos esta lista, eliminando primero los mencionados por un porcentaje mínimo de sujetos y luego agrupando los sinónimos a fin de evitar repetición de significados. 
Junto a estos adjetivos decidimos incluir algún adjetivo que gozaba de una elevada frecuencia de atribución (por sujetos gallegos) en el etudio de Sangrador (amantes de su tierra, trabajadores, inteligentes, materialistas, ambiciosos, tradicionalistas). Finalmente se buscó un antónimo para cada uno de los 19 adjetivos seleccionados, confeccionando así las escalas bipolares ya mencionadas.

\section{RESULTADOS}

\section{Elección e interpretación de la dimensionalidad en la muestra total}

Inicialmente se realizó un Escalamiento Multidimensional de los mencionados estímulos en orden a conocer las dimensiones subyacentes a la percepción ciudadana de los diferentes estereotipos señalados. Para ello, se utilizó el programa ALSCAL de Takane, Young y Deleeuw (1977). Los datos de entrada fueron 86 matrices simétricas de disimilaridad, de orden $7 \times 7$, una para cada sujeto. Solamente se utilizó la información contenida en el triángulo inferior de cada matriz. Siguiendo la terminología sugerida por Carroll y Arabie (1980) el nivel de medida supuesto fue ordinal, el proceso discreto, la matriz condicional y el modelo con replicación. Se obtuvieron soluciones en $5,4,3,2$, y 1 dimensiones.

Posteriormente, con el fin de seleccionar las dimensiones subyacentes a la representación espacial de estímulos ofrecida por el MDS y de facilitar su interpretación, se realizaron varios análisis de regresión lineal múltiple con el programa P1R del sistema BMDP. Para ello, se utilizaron como variables dependientes las puntuaciones medias de todos los sujetos en las 19 escalas bipolares, para cada uno de los estímulos considerados y, como variables independientes las coordenadas de los estímulos ofrecidas por el MDS.

Combinando los resultados obtenidos por estos dos análisis mencionados, se seleccionó la solución en dos dimensiones. La Figura 1 muestra la configuración de estímulos ofrecida por el MDS en un espacio bidimensional (Stress $=396$ ) (Young y Lewychyj, 1979).

Observamos que algunos estímulos aparecen próximos en el espacio, como por ejemplo: gallegos-vascos, catalanes-europeos y andaluces-españoles. Suponemos que dicha proximidad de los estímulos en el espacio está relacionada con algunas propiedades de éstos que nos permitirían explicar la percepción por parte de los individuos de dichos grupos como más similares.

En la Tabla II se ofrecen los coeficientes de correlación y regresión entre las coordenadas de puntos derivadas por el Escalamiento Multidimensional en las dimensiones I y II y cada una de las escalas bipolares. Estos valores, ofrecidos por el análisis de regresión, nos permitirían interpretar las dimensiones subyacentes a la representación espacial de estímulos ofrecida por el MDS. Una correlación alta y significativa nos indicaría que esa escala puede ser identificativa de una de las dimensiones, si además el coeficiente de regresión es alto y significativo en esa dimensión (Kruskal y Wish, 1978).

Así, se ha obtenido que los pesos más altos en la dimensión I corresponden a las escalas bipolares inteligentes -no inteligentes $(.900, \mathrm{p}<.01)$ y responsables - irresponsables $(.852, \mathrm{p}<.05)$. Trasladando estos resultados a la Figura 1 , podemos decir que esta dimensión está dominada por los catalanes (en el polo positivo) y los andaluces (en el negativo). Ello nos permitiría interpretar 
Figura 1

Representación de estímulos en la muestra total. Modelo con replicación en 3 vías $(N=86)$

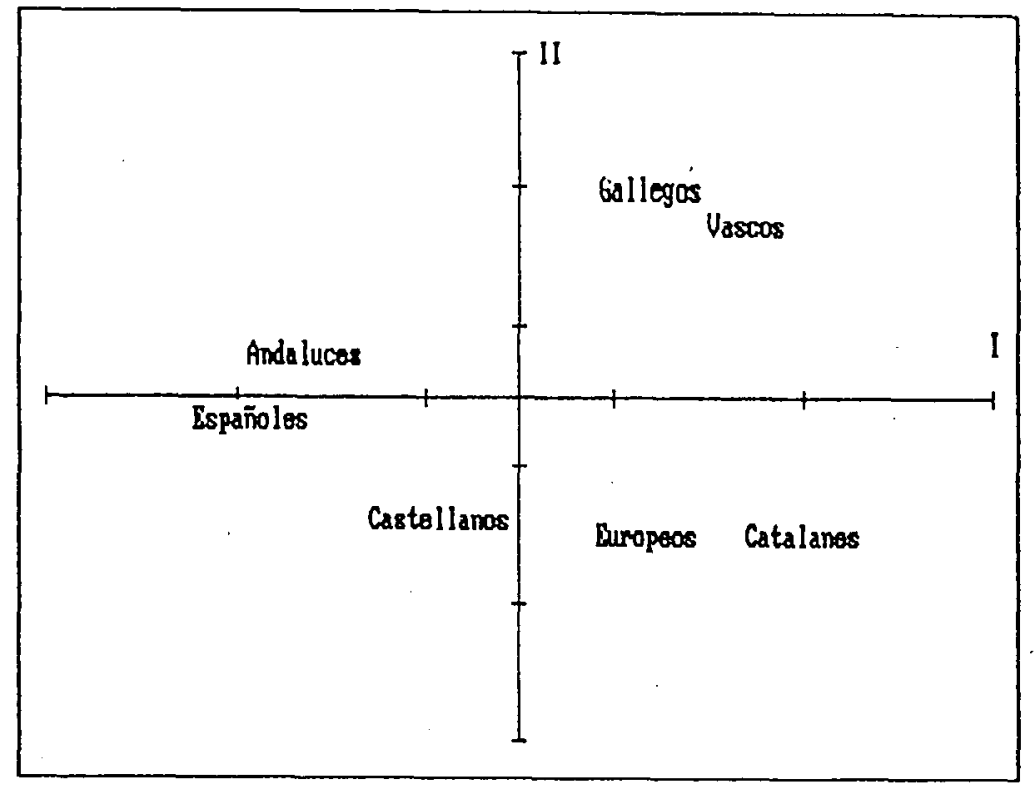

TABLA II

Coeficientes de regresión tipificados y coeficientes de correlación entre las coordenadas de puntos ofrecidas por el programa MDS y cada una de las escalas bipolares (muestra total $N=86$ )

\begin{tabular}{|c|c|c|c|}
\hline \multirow[t]{2}{*}{ Escala } & \multicolumn{2}{|c|}{ Coef. Regresión } & \multirow[t]{2}{*}{ Corr. Múlt. } \\
\hline & I & II & \\
\hline Pacíficos & -.330 & .200 & .386 \\
\hline Tolerantes & -.605 & -.067 & .6085 \\
\hline Generosos & $-.596 \%$ & $-.666^{* \%}$ & $.8934^{* * *}$ \\
\hline Sinceros & .131 & -.610 & .6242 \\
\hline Confiados & $-.798 \%$ & -.029 & .7988 \\
\hline Responsables & $.852 *$ & .160 & $.8670 \%$ \\
\hline Tradicionales & $-.562^{* \cdots *}$ & $-.796^{* * *}$ & $9737 \div$ \\
\hline Sensibles & -.588 & -.509 & .7768 \\
\hline Rígidos & $.800 *$ & .125 & .8091 \\
\hline Arrogantes & .407 & .623 & .7433 \\
\hline Solidarios & -.478 & $-.779 \%$ & $.9131 \%$ \\
\hline Idealistas & -.361 & $-.766 \%$ & .8458 \\
\hline Agradables & $-.657 * \%$ & $-.598 *$ & $.8870 \div \div$ \\
\hline Próximos & $-.735^{* *}$ & $-.528 \%$ & $.9037 *$ \\
\hline Impulsivos & -.537 & -.543 & .7631 \\
\hline Ambiciosos & .632 & .551 & .8377 \\
\hline Amant. tierra & .265 & -.624 & $: 6783$ \\
\hline Inteligentes & $.900 \%$ & .236 & $.9301 \%$ \\
\hline Trabajadores & $.839 \%$ & .118 & .8471 \\
\hline
\end{tabular}

Siginificación estadística: $\quad *<.06$

$* *<.05$

$\because \%<.01$ 
la dimensión como «Competencia Personal» ya que los atributos"que más pesan sobre esta dimensión se refieren a características personales de los sujetos evaluados.

Con respecto a la dimensión II, las escalas con mayor peso fueron tradicionales-modernos $(-.796, \mathrm{p}<.01)$ y solidarios-insolidarios $(-.779, \mathrm{p}<.01)$, teniendo las demás escalas pesos más bajos. Trasladando estos resultados a la Figura 1 (eje vertical), gallegos y vascos serían percibidos como más tradicionales y solidarios frente a los estereotipos catalanes y europeos. A tenor de los adjetivos con un mayor peso en esa dimensión, ésta podría denominarse «valores sociales».

Por otra parte, esta dimensionalidad de los estereotipos se vería confirmada, posteriormente, por un análisis de frecuencias realizado para cada escala y estímulo. Así, de forma paralela, observabamos que los adjetivos que definín características personales (trabajadores, inteligentes, responsables, rígidos, ambiciosos) situaban en polos opuestos, respecto al eje horizontal, a catalanes y europeos frente a andaluces, y en menor medida respecto a castellanos y españoles. Respecto a la dimensión II serían los atributos de carácter más social (tradicionales, solidarios, idealistas, sinceros) los que diferenciarían a los estereotipos gallegos y vascos con respecto a los catalanes y europeos. Adicionalmente, pudimos observar que serían los sujetos castellanos los más ambiguamente definidos por los sujetos gallegos, en el sentido de una mayor dispersión de las respuestas, así como en la emisión de respuestas intermedias en la escala discreta de 9 puntos. Ello nos hizo pensar en la incertidumbre por parte de los sujetos al evaluar a dicho grupo, quizás por no estar tan claramente identificado con una determinada región autonómica o, por su similitud con el grupo de españoles. Algo muy similar observabamos respecto a los sujetos europeos, aunque suponemos que por otras razones como la gran cantidad de países que abarcan y en consecuencia la ausencia, por parte de los sujetos gallegos, de una imagen estereotipada que los caracterice a todos ellos.

Adicionalmente, con fines confirmatorios, se llevó a cabo un nuevo análisis utilizando como entrada la media aritmética de las 86 matrices de disimilaridad originales. Ello nos permitiría comparar la configuración de estímulos ofrecida por el modelo con replicación con datos de entrada en 3 vías (estímulos $\mathrm{x}$ estímulos $\mathrm{x}$ sujetos), representada en la Figura 1 , con la solución en 2 vías (estímulos $\mathrm{x}$ estímulos). La representación obtenida de los estímulos en el plano fué muy similar a la ofrecida en la Figura 1.

Otro modelo de Escalamiento Multidimensional utilizado permitiría confirmar los hallazgos encontrados: el INDSCAL (véase, p. e., Arce y Gärling, 1989; Carroll y Chang, 1970; Arabie, Carroll y DeSarbo, 1987). A diferencia del modelo con replicación, el INDSCAL (modelo de diferencias individuales) nos permitiría, además de obtener una representación de estímulos, una configuración de los sujetos. Por lo que podemos destacar, con independencia del modelo de MDS utilizado, la consistencia de los datos en la representación espacial de los 7 estereotipos estudiados.

\section{Ponderación de las dimensiones en las variables sexo y profesión}

Una vez elegida e interpretada la dimensionalidad en la muestra total se realizaron dos análisis estadísticos más. 
En primer lugar, se realizó un nuevo MDS en orden a conocer si los sujetos de ambos sexos ponderaban de igual manera en las dimensiones obtenidas. El modelo elegido para el análisis fue el INDSCAL, que nos ofrecería a la vez que la representación de los estímulos, la representación en el espacio del grupo de hombres y mujeres. Para ello se consideraron las dos siguientes submuestras: una de universitarios hombres $(\mathrm{N}=15)$ y otra de universitarios mujeres $(\mathrm{N}=31)$. Los datos de entrada estaban formados por dos matrices simétricas, de orden $7 \times 7$, medias aritméticas de las matrices de disimilaridad originales en ambas muestras. El nivel de medida fue de intervalo, el proceso contínuo y la matriz incondicional. La configuración de estímulos, en dos dimensiones, es muy similar a la solución obtenida para la muestra total. El valor de SStress fue de .134, lo que nos permite concluir un buen ajuste entre el modelo y los datos.

Por otra parte, la representación en el plano del grupo de hombres y mujeres nos permitiría ver las posibles diferencias entre ellos. Suponemos que cuanto menor sea el ángulo que describen los vectores para cada uno de los grupos menor serán las diferencias entre ellos y mayor cuanto más grande sea. En base a ello la Figura 2 nos muestra como hombres y mujeres describen un ángulo pequeño, que denotaría pequeñas diferencias entre ellos.

FIGURA 2

Configuración espacial de hombres y mujeres. Modelo INDSCAL.

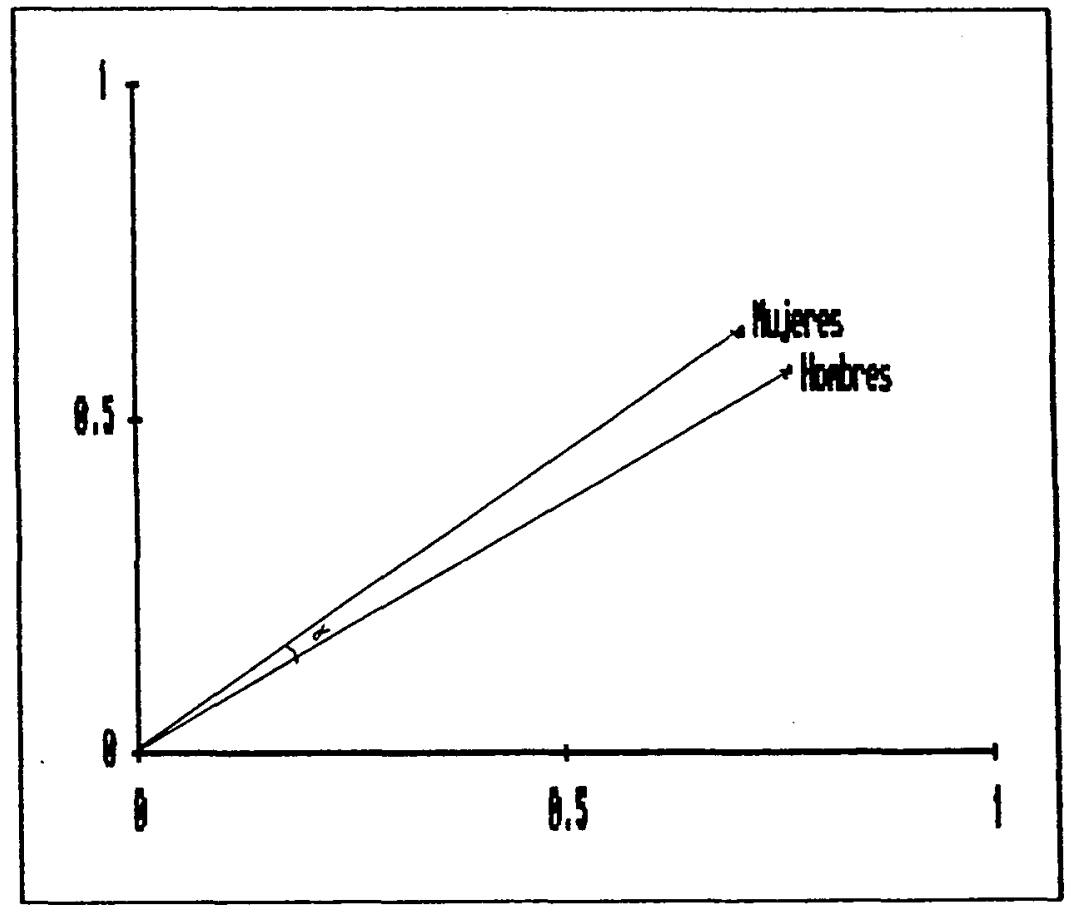

De todo lo expuesto hasta el momento, podemos señalar que, para las muestras objeto de estudio, no hay diferencias marcadas entre hombres y mujeres respecto a la percepción de los diferentes estereotipos y a las dimensiones subyacentes a ésta. 
Esto no es extraño, si tenemos en cuenta que las escasas investigaciones, que han tenido en cuenta esta variable, concluyen en la no existencia de diferencias entre hombres y mujeres (Sinha y Upadhyaya, 1960; Zaidi, 1967; etc.). Asimismo, utilizando una muestra de sujetos españoles, Mateos y Aberrasturi (1961) encontraron una correlación aproximada de 0,90 entre las preferencias nacionales de ambos sexos.

En segundo lugar, se llevó a cabo una nueva aplicación del modelo INDSCAL con el fin de observar las posibles semejanzas o diferencias en la representación espacial de estímulos ofrecida por éste en base a la variable profesión. Las submuestras consideradas fueron dos: una primera muestra de estudiantes $(\mathrm{N}=46)$ y una segunda de trabajadores $(\mathrm{N}=40)$. De modo similar al análisis anterior, los datos de entrada fueron dos matrices simétricas, medias aritméticas de las puntuaciones originales. Los parámetros considerados fueron los mismos que en el análisis anterior.

Los resultados obtenidos, en dos dimensiones, para las dos muestras tanto por lo que respecta a la agrupación de los estímulos, como a las diferencias entre las muestras. En este caso, el nivel de Stress fue de .192. Esta similitud, nos permite suponer, para el presente estudio, que las dimensiones subyacentes a la percepción de los diferentes grupos nacionales y regionales con las mismas a las encontradas hasta el momento, independientemente del sexo o de la profesión de los sujetos experimentales. Así, la dimensión I correspondería a atributos personales de los grupos estereotipados, mientras que la dimensión II se relacionaría con atributos sociales.

\section{Análisis de clusters}

Además de las dimensiones encontradas en las representaciones de estímulos ofrecidas por el Escalamiento Multidimensional para las distintas muestras consideradas, se observó que también existían importantes agrupaciones de los estímulos en cada uno de los cuadrantes. Precisamente en orden a conocer mejor estas pequeñas estructuras se realizó un análisis de cluster, con el fin de observar qué estereotipos se agrupaban en función de su similitud (Arabie, Carroll y De Sarbo, 1987). Los datos de entrada para cada análisis, fueron las matrices medias de disimilaridad de las muestras estudiadas. Para ello se utilizó el paquete estadístico SPSS/PC+. El método empleado para agrupar los estímulos fue el análisis de grupo jerárquico aglomerativo. En éste, las agrupaciones se forman uniendo estímulos en grupos cada vez mayores hasta que todos los estímulos son miembros de una sola agrupación. Por otra parte, con el fin de conocer, en la medida de lo posible el ajuste entre el modelo y los datos se aplicaron dos métodos de agrupamiento: el método de vínculo único (single link) y el método de vínculo completo (complete link). Suponemos que cuanto más similares sean los resultados aportados por ambos métodos, mejor será el ajuste y peor, cuanto más distintos sean. En ambos casos, los dos primeros casos combinados son aquellos que guardan la mayor similizud entre sí o la menor distancia. En el método de vínculo único, un estímulo se unirá a un cluster ya existente sólo si en dicho grupo alguno de los estímulos está en el mismo nivel de similaridad que éste (Sneath, 1957). En el método de vínculo completo, para que un estímulo se una a un cluster tendrá que estar dentro de un cierto nivel de similaridad con los estímulos que forman ya el cluster (Sokal y Michener, 1958). 
Dada la gran semejanza, por una parte de representaciones de estereotipos en las distintas muestras estudiadas, y por otra, de soluciones obtenidas a través de los dos métodos de agrupamiento utilizados, sólo presentaremos el cluster de estereotipo encontrado para la muestra total por medio del método de vínculo completo. En la Figura 3 se ofrece dicha solución.

\section{Figura 3}

Cluster de estereotipos para la muestra total. Método de vinculo completo.

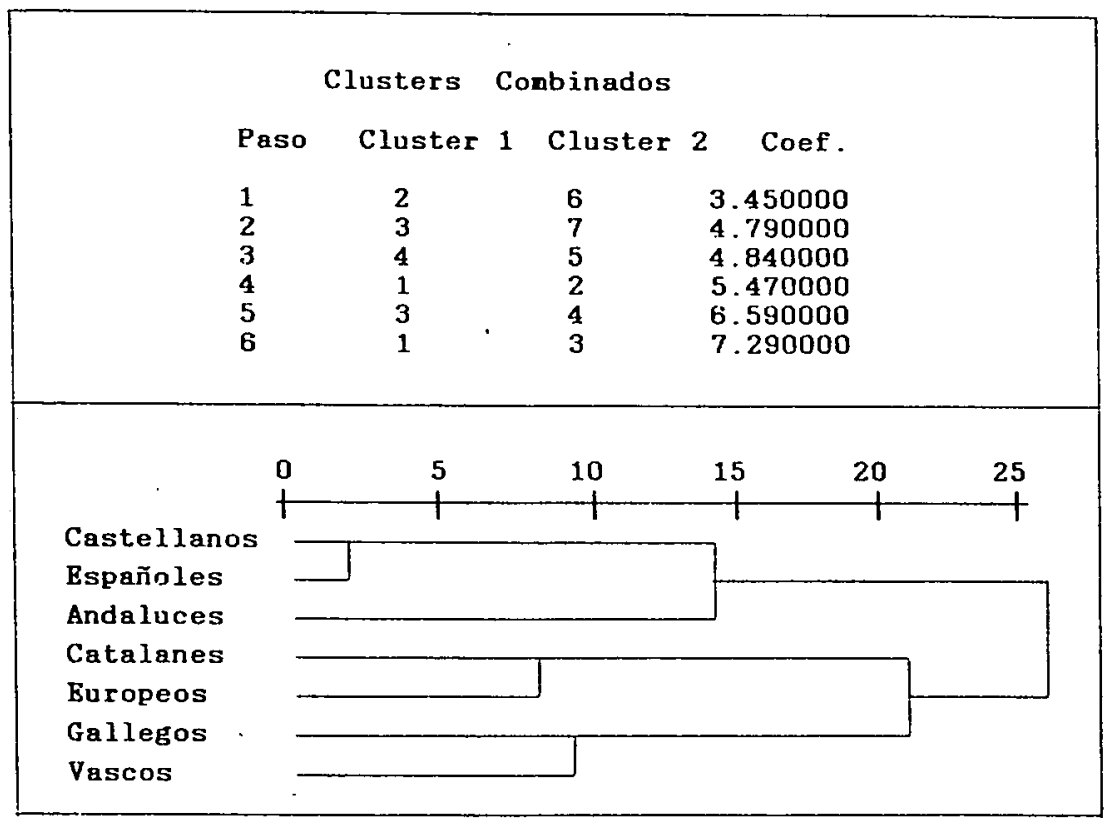

Podemos observar como, en un primer paso, se unirían castellanos y españoles como grupos más similares. Con una distancia algo mayor estaría el segundo cluster formado por catalanes y europeos, y en un tercer paso aparecerían formando cluster gallegos y vascos. Finalmente, andaluces se agruparía al primer cluster formado por castellanos y españoles, y estos al segundo y tercer cluster.

De este modo, el análisis de cluster realizado nos permitiría confirmar la existencia real de agrupaciones de los estereotipos que habíamos visualizado en la configuración espacial de estímulos ofrecida por el Escalamiento Mulridimensional:

\section{Representación política de los estereotipos}

La similitud de resultados encontrados hasta el momento para las variables estudiadas nos llevó a considerar, en una segunda fase del estudio, el análisis de una tercera variable (i. e. actitud política) que suponíamos podría marcar diferencias en la percepción ciudadana de los estereotipos.

Todos los estudiosos del tema coinciden en señalar que las actitudes hacia determinados grupos están marcadas por las relaciones intergrupales, a la vez 
que determinados factores políticos, económicos, históricos tienen la posibilidad de cambiarlos. En base a ello, pensamos que la identificación de los sujetos con una determinada ideología política, serviría al estudio de las diferencias en la percepción de los diferentes grupos nacionales y regionales objeto de estudio.

Por otra parte; la orientación política de los sujetos, tal como se muestra en un reciente trabajo de Sabucedo y Arce (1990), se relaciona con diferentes grados de sensibilidad hacia el hecho nacionalista y la identidad nacional. Así, en ese estudio se observó como los sujetos que se autoidentificaban con una posición política de izquierdas manifestaban, en mayor medida que los de derecha, su deseo de que Galicia alcanzase cotas más altas de autogobierno, y se autodefinían como más gallegos que españoles.

Un análisis de Escalamiento. Multidimensional, con muestras de sujetos de izquierdas y derechas, nos permitiría contemplar este tercer objetivo. Para ello, se utilizó el Modelo INDSCAL. La entrada en el análisis estaba formada por dos matrices simétricas, de orden $7 \times 7$, medias aritméticas respectivamente de las matrices de disimilaridades originales para los sujetos de izquierdas $(\mathrm{N}=37)$ y de derechas $(\mathrm{N}=14)$. El nivel de medida fue de intervalo, el proceso contínuo y la matriz incondicional. En contra de lo que suponíamos, la solución obtenida (SStress $=.237$ ) no muestra diferencias marcadas respecto a la representación cognitiva de los estímulos encontrada hasta el momento. Por otra parte la representación, en el plano bidimensional, de los dos grupos ideológicos, confirmaría los resultados encontrados, observando como la diferencia entre sujetos de izquierdas y derechas es pequeña.

\section{CONCLUSIONES}

El objetivo de este trabajo consistía en descubrir las dimensiones subyacentes a la percepción que los sujetos gallegos tenían de diferentes regiones y nacionalidades. A diferencia de otros estudios, el nuestro pretendía ir más allá de una simple recogida de atributos que unos grupos utilizan para evaluar y juzgar a otros. Por ello, recurrimos a la utilización de un modelo de distancia espacial, el escalamiento multidimensional que nos permitía estudiar la distancia psicológica entre los diferentes grupos objeto de estudio en la configuración espacial ofrecida por ese modelo. De acuerdo con Sabucedo y Godás (1985) esta técnica de análisis resulta muy adecuada y pertinente para el tratamiento de diferentes cuestiones psicosociales.

Los resultados obtenidos, nos muestran la existencia de dos dimensiones a la hora de valorar los distintos grupos-estímulo con los que hemos trabajado. La primera de las dimensiones viene definida fundamentalmente por los adjetivos inteligente-no inteligente y responsables-irresponsables. La segunda por los adjetivos tradicionales-modernos y solidario-insolidario. A estas dos dimensiones las hemos denominado competencia personal y valores sociales, respectivamente.

De acuerdo con los datos obtenidos, los gallegos se perciben próximos a los vascos, evalúan a catalanes y europeos de forma similar, y consideran a los españoles, castellanos y andaluces formando parte de un mismo grupo. Por otra parte, el grupo de gallegos y vascos se hallan más próximos al de catalanes y europeos que al de castellanos, andaluces y españoles. 
Al margen de lo anterior, hay otro aspecto en el presente estudio, al que hemos aludido en la introducción a este trabajo, que tiene suma relevancia en cuanto que incide en uno de los grandes temas de debate en torno al concepto y definición de los estereotipos. Nos referimos al hecho de la estabilidad mostrada en la relación entre las dimensiones y los grupos estudiados. Esa relación se mantiene a través de las distintas submuestras utilizadas en nuestro estudio: orientación política de derechas versus izquierda, universitariosno universitarios, hombres-mujeres; y de los diferentes modelos de análisis empleados. Esto indicaría que nos encontramos ante unas creencias estables y consolidadas y asumidas por sectores muy diferentes de población. El hecho de que variables como sexo, orientación política, tipo de estudios no presenten diferencias en la evaluación de los distintos estímulos presentados, avala esa interpretación.

Es posible que si se utilizasen otros grupos de sujetos, p. ej. sujetos con una marcada ideología nacionalista, o si se manipulasen las situaciones de interacción entre los grupos, del modo en que lo hizo Sherman (1973) en su estudio sobre naciones, los resultados podrían presentar ciertas discrepancias entre las muestras estudiadas. Sin embargo, eso no iría en contra de la tesis del carácter consensual de los estereotipos. Antes al contrario, esas creencias diferentes reflejarían el tipo de relaciones intergrupales existentes, y, por ello, serían compartidas por los miembros de esos grupos. Por otra parte, si abandonamos la aproximación perceptual, característica de los estudios sobre categorización, y adoptamos una aproximación argumentativa y retórica (Billig, 1985), deberíamos reconocer la posibilidad de coexistencia de creencias muy diferentes y compartidas en el discurso social sobre distintos grupos.

Los resultados obtenidos, y la constatación de ese consenso a la hora de valorar distintos grupos humanos, nos permite un mayor conocimiento de la génesis y el mantenimiento de los estereotipos. En este caso, parece obvio que procesos cognitivos como el de la categorización no pueden dar cuenta por sí solos de la naturaleza y alcance de los fenómenos que estamos estudiando. El propio Tajfel (1984) había apuntado ese mismo hecho. Hay que ir más allá de los simples procesos cognitivos y vincular los estereotipos a factores sociales e históricos que son los que están dando sentido y explicando el discurso que se mantiene respecto a otros grupos. Sin el recurso a este tipo de dimensiones cualquier intento de comprensión de la problemática de los estereotipos resultará infructuoso. Porque no se trata simplemente que los sujetos diferencien ciertos grupos, sino que les atribuyan unos rasgos y unas características y no otras. $Y$ esto se realiza al margen de que exista un conocimiento directo de esos grupos y de forma compartida.

El carácter consensual y compartido de esas creencias nos indica que las mismas no son individuales, sino que tienen un carácter claramente social. De este modo, frente a las tesis que mantienen una aproximación individualista a los fenómenos psico-sociales, habría que resaltar la dimensión social del conocimiento. Esto no significa únicamente que las variables sociales y culturales están afectando el modo en que se percibe la realidad, algo que họoy en día nadie cuestiona. Lo que queremos decir, y lo que los datos de nuestro estudio parecen apoyar es que existe un discurso social, un sentido común que determina el modo de ver y entender la realidad, y que por ello son parte de esa misma realidad (Moscovici, 1984, Ibañez, 1988).

En definitiva, el análisis de los estereotipos no debe de realizarse desde 
posiciones que cómo la cognitiva implican un reduccionismo individualista y una consideración estática y burocratizada del pensamiento humano (Billig, 1985, 1987, Billig et al. 1988). Los estereotipos, al igual que otros fenómenos psicosociales se instalan en una dimensión șocial. Atendiendo a este nivel de análisis, muchas de las variables tradicionalmente utilizadas en psicología social, como motivaciones, aspiraciones y principios cognitivos pierden gran parte de la relevancia que hasta ahora se le había concedido (Moscovici, 1984, p. 65), a la vez que los llamados sesgos en el pensamiento y percepción adquieren un nuevo significado. Estos ya no pueden ser considerados como distorsiones cognitivo-individuales, sino que son parte del sentido común y de las representaciones sociales existentes en un momento determinado.

Por otra parte, el situar a los estereotipos en el terreno del discurso y de las representaciones sociales, y no mantenerlo vinculado exclusivamente a procesos como el de la categorización, permite cierta posibilidad de solución al dilema planteado por Hamilton (1979) según el cual el prejuicio sería inevitable. Si los estereotipos y los prejuicios son discursos sociales, es posible elaborar otros discursos y sentido comunes que se opongan al anterior. En esa tarea la psicología social debe jugar un papel importante.

\section{Referencias}

AlLPORT, G. W. (1954). La naturaleza del prejuicio. Buenos Aires: EUDEBA, S. A.

ArabiE, P., Carroll, J. D., y DeSArbo, W. (1987). Three-way scaling and clustering. Newbury Park. CA: Sage.

ArCe, C. (1989a). Tres notas sobre el análisis de datos en psicología. En Cognición e Inteligencia. Libro homenaje al profesor Yela. En preparación.

Arce, C. (1989b). Escalamiento Multidimensional. En J. Arnau (Comp.), Métodos y Técnicas de Análisis de datos en Ciencias del Comportamiento. En preparación.

ArCe, C., y Gärling, T. (1989). Multidimensional Scaling. Anuario de psicología, 43, en prensa.

Arce, C., Seoane, G., y Varela, J. (1989). Modelos básicos en Escalamiento Multidimensional. Psicológica, 10, 35-48.

BILlig, M. (1985). Prejudice, categorization and particularization: From a perceptual to a rhetorical approach. European Journal of Social Psychology, 15, 79-103.

Billig, M. (1987), Arguinig and Thinking. A Rbetorical approach to social Psychology. Cambridge, NJ: Cambridge University Press.

Billig, M. et al. (1988). Ideological Dilemmas. Newbury Park: Sage.

Brigham, J. C. (1971). Ethnic Stereotypes. Psychological Bulletin, 76, 15-38.

Caplan N., y Nelson, S. D. (1973). On Being useful: The nature and consequences of Psychological research on social problems. American Psychologist, 28, 199-211.

Carol, J. D., y Arabie, P. (1980). Multidimensional Scaling. En M. R. Rosenzweig, y L. W. Porter (Comp.), Annual Review of Psychology, (Vol. 31, pp. 607-649). Palo Alto, CA: Annual Reviews.

CAROL, J. D., y ChANG, J. J. (1970). Analysis of individual differences in multidimensional scaling via N-way generalization of Eckart-Young decomposition. Psychometrika, 35, 283-319.

Chacon Fuertes, F. (1986). Estereotipos regionales madrileños. Papeles del Colegio. Revista del Colegio Oficial de Psicólogos, Vol. 4, 25, 23-30.

Davison, M. L. (1983). Multidimensional Scaling. Nueva York: Wiley.

GILBERT, G. M. (1951). Stereotype persistence and change among college studentes. Journal of Abnormal \& Sacial Psychology, 46, 245-254.

Hamilton, D. L. (1979). A cognitive-attributional analysis of stereotyping. Advances in Experimental Social Psychology, 23, 53-84.

Hamilton, D. L. (1981) Cognitive Processes in Stereotyping and Intergroup Bebavior. Hillsdale: L. E. A.

HAMILTON, D. L., y GuIFFORD, R. K. (1976). Illusory correlations in interpersonal perception: A cognitive basis of stereotypic judgements. Joumal of Experimental Social Psychology, $12,392-407$.

IbAÑez, T. (1988). Ideologías de la Vida Cotidiana. Barcelona: Sendai Ediciones. 
Kari.ins, M., Coffman, T. L., \& Walters, G. (1969). On the fading of social stereotypes: Studies in three generations of college students. Journal of Personality \& Social Psychology, $13,1-16$

KATZ, D. (1974). Factors Affecting Social Change: A Social-Psychological Interpretation. Journal of Social Issues, 30, 3, 159-180.

KATZ, D. y BRALY, K. (1933). Racial Stereotypes of one hundred students. Journal of Abnormal E Social Psychology, 28, 280-290.

Kruskal, J. B., y W1SH, M. (1978). Multidimensional Scaling. Newbury Park, CA: Sage.

Lippmann, W. (1982). Public Opinion. New York: Mc. Millan.

MACKIE, M. M. (1973). Arriving at truth by definition: Case of stereotype innacuracy. Social Problems, 20, 431-447.

Mateos M., y Aberrasturri, N. L. (1961). Nuevas aportaciones al estudio de las preferencias nacionales en España. Revista de Psicología General y Aplicada, 57, 49-65.

MEENES, M. (1943). A comparison of racial stereotypes of 1935 and 1943. Journal of Social Psychology, 17, 327-336.

Moscovicl, S. (1982). The coming era of representations. En J. P. Codol y J. J. Leyens (Eds): Cognitive Approaches to Social Behavior. The Hague: Nijhoff.

Moscovicl, S. (1984). The phenomenon of social representations. En R. M. Farr, y S. Moscovici (comp.), Social Representations. Cambridge, Cambridge University Press.

Pinil.Los, J. L. (1960). Estereotipos raciales de universitarios españoles, ingleses y norteamericanos. Revista de Psicología General y Aplicada, 56, 777-797.

Rodriguez Sanabra, F. (1963). Estereotipos regionales españoles. Revista de Psicología General y Aplicada, 68-69, 763-771.

SABucrdo, J. M, y ARCE, C. (1990). Actitudes sociales y comportamiento político de la población juvenil gallega. Investigación subvencionada por la Dirección General de Ordenación Universitaria y Política Científica (D. O. G. 9-3-89).

Sabuckido, J. M, y Godas, A. (1985). Métodos y Técnicas de investigación en Psicología Social. Santiago: Tórculo.

Sabucedo, J. M, y Rodricuez, M. (1990). Racionalidad y dimensión social de la acción política. Boletín de Psicología, 27, 55-70.

SANGRADOR, J. L. (1981). Grupos bumanos y categorías sociales. Barcelona: Herder.

Sangrador, J. L. (1990). Estereotipos sociales: notas sobre sus rasgos definitorios. III Congreso Nacional de Psicología Social.

SHERman, R. C. (1973). Dimensional salience in the perception of nations as a function of attitudes toward war and anticipated social interacrion. Journal of Personality and Social Psychology, 27, 65-73.

Sinha, A. K. P., y Upadhyaya, O. P. (1960). Stereotypes of male and female university students in India toward different ethnic groups. Journal of Social Psycology, 51, 93-102.

SNI:ATH, P. (1957). The applications of computers to taxonomy. Journal of General Microbiology, $17,201-226$.

SoKAl., R. y MiCHENiR, C. D. (1958). A statistical method for evaluating systematic relationships. University of Kansas Scientific Bulletin. 38, 1409-1438.

TAjFil, H. (1975). La categorización social. En S. Moscovici (Comp.), Introducción a la Psicología Social (Vol. 8, pp. 349-387). Barcelona: Planeta.

TAJPE:, H. (1984). Grupos bumanos y categorías sociales. Barcelona: Herder.

TAJPl:, H., y WILKE:S, A. L. (1963). Classification and quantitative judgement. British Journal of Psychology, 54, 101-114.

Takanl:, Y., YOUNG, F. W., y Deleeuw, J. (1977). Nonmetric individual differences multidimensional scaling: an alternating least method wich optimal scaling features. Psychometrika, 17, 401-419.

TAYloR, D. M., y ABoud, F. E. (1973). Ethnic stereotypes: Is the concept necessary?. Canadian Psychologist, 14, 330-338.

Tayior, S. E., Fiske, S. T., Etcoff, N. L., y Ruderman, A. (1978). Categorical and contextual bases of person memory and stereotyping. Journal of Personality and Social Psychology, 36, 778-793.

VINACK1:, W. E. (1956). Stereotypes as social concepts. Journal of Social Psychology, 46, 229243.

Younc, F. W., y LewyckyJ, R. (1979). Alsal. User's Guide. 3rd. edition. Chapel Hill: Data Analysis and Theory Associates.

ZAIDI, S. M. H. (1967). A study social distance as perceived by students of Karachi university. Journal of Social Psychology, 71, 197-207. 